\title{
Absence of superconductivity in iron polyhydrides at high pressures
}

\author{
Christoph Heil, ${ }^{1,2,{ }^{*}}$ Giovanni B. Bachelet, ${ }^{3}$ and Lilia Boeri ${ }^{3}$ \\ ${ }^{1}$ Department of Materials, University of Oxford, Parks Road, Oxford OX1 3PH, United Kingdom \\ ${ }^{2}$ Institute of Theoretical and Computational Physics, Graz University of Technology, NAWI Graz, 8010 Graz, Austria \\ ${ }^{3}$ Dipartimento di Fisica, Sapienza Università di Roma, 00185 Roma, Italy
}

(Received 10 April 2018; revised manuscript received 17 May 2018; published 13 June 2018)

\begin{abstract}
Recently, C. M. Pépin et al. [Science 357, 382 (2017)] reported the formation of several new iron polyhydrides $\mathrm{FeH}_{x}$ at pressures in the megabar range and spotted $\mathrm{FeH}_{5}$, which forms above $130 \mathrm{GPa}$, as a potential high- $T_{\mathrm{c}}$ superconductor because of an alleged layer of dense metallic hydrogen. Shortly after, two studies by A. Majumdar et al. [Phys. Rev. B 96, 201107 (2017)] and A. G. Kvashnin et al. [J. Phys. Chem. C 122, 4731 (2018)] based on ab initio Migdal-Eliashberg theory seemed to independently confirm such a conjecture. We conversely find, on the same theoretical-numerical basis, that neither $\mathrm{FeH}_{5}$ nor its precursor, $\mathrm{FeH}_{3}$, shows any conventional superconductivity and explain why this is the case. We also show that superconductivity may be attained by transition-metal polyhydrides in the $\mathrm{FeH}_{3}$ structure type by adding more electrons to partially fill one of the $\mathrm{Fe}-\mathrm{H}$ hybrid bands (as, e.g., in $\mathrm{NiH}_{3}$ ). Critical temperatures, however, will remain low because the $d$-metal bonding, and not the metallic hydrogen, dominates the behavior of electrons and phonons involved in the superconducting pairing in these compounds.
\end{abstract}

DOI: 10.1103/PhysRevB.97.214510

\section{INTRODUCTION}

The discovery of a record superconducting $T_{\mathrm{c}}$ of $203 \mathrm{~K}$ in $\mathrm{H}_{3} \mathrm{~S}$ has confirmed Ashcroft's 15-year-old suggestion that hydrogen-dominant metallic alloys are good candidates for the high- $T_{\mathrm{c}}$ conventional superconductivity at lower pressure than the one needed to turn molecular hydrogen into a metallic superconductor (as predicted by Ashcroft 50 years ago) [1-3]. This has ignited an intense theoretical and experimental search for new superconductors at high pressures, and presently, three groups of materials seem to stand out: (i) hydrogen itself, in its high-pressure molecular (insulating) and atomic (metallic) phases [4-6]; (ii) covalent hydrides, which form molecular solids at normal pressure and crystalline metals at high pressure [7-13]; and (iii) heavy-metal hydrides with open hydrogen cages (involving $\mathrm{Ca}, \mathrm{Y}, \mathrm{La}$, U, etc.), which form at $P \gtrsim 200 \mathrm{GPa}$ [14-16]. All of them are unusual metals with strongly directional bonds [17], yielding a large electron-phonon (e-ph) coupling, but each requires extreme stabilization pressures. Hence, the challenge in this field of research is to devise chemical strategies to obtain lower and lower formation pressures for such high- $T_{\mathrm{c}}$ conventional superconductors [18] by identifying (or ruling out) classes of plausible candidates and electronic bands relevant for their $e$-ph interaction.

Recently, Pépin et al. [19] reported the synthesis of a new iron hydride at $130 \mathrm{GPa}$ : the hydrogen-rich layered crystal $\mathrm{FeH}_{5}$, a metal which seems like a promising candidate for high- $T_{\mathrm{c}}$ superconductivity; shortly after, two theoretical papers argued that $\mathrm{FeH}_{5}$ should indeed exhibit $T_{\mathrm{c}}$ 's as high as $56 \mathrm{~K}$ [20,21]. In this work, using ab initio Migdal-Eliashberg theory as implemented in the EPW code [22], we show that the $T_{\mathrm{c}}$ of

*christoph.heil@tugraz.at
$\mathrm{FeH}_{5}$ is actually $\leqslant 1 \mathrm{~K}$ and that for this compound the picture of a dense two-dimensional metallic-hydrogen layer is not sensible; on the contrary, $\mathrm{FeH}_{5}$ bears a very strong resemblance to its precursor, $\mathrm{FeH}_{3}$, which is also not superconducting. In both compounds the conductivity is dominated by $d$-metal bonding, which, under appropriate circumstances, may yield conventional superconductivity but not high $T_{\mathrm{c}}$.

\section{RESULTS AND DISCUSSION}

According to Pépin et al. [19], $\mathrm{FeH}_{5}$ forms above $130 \mathrm{GPa}$ by hydrogenation of $\mathrm{FeH}_{3}$, which is stable between 85 and $130 \mathrm{GPa}$. The structure was experimentally determined by measuring the x-ray diffraction patterns and performing a Rietveld refinement. Due to the low scattering power of the hydrogen atoms, only the $\mathrm{Fe}$ positions could be measured experimentally, and a structural search with density functional theory was employed by Pépin et al. to find the lowest-enthalpy structure that is in agreement with both $\mathrm{x}$-ray diffraction patterns and volume vs pressure curves from experiment [19]. Our own evolutionary structure searches with USPEX [23] confirm that the proposed structures for $\mathrm{FeH}_{3}$ and $\mathrm{FeH}_{5}$ are indeed stable in the considered pressure ranges $[19,24]$. We depict the two crystal structures in Fig. 1 and, in the following, will consider both compounds at the same pressure of $150 \mathrm{GPa}$. The computational details of all our calculations are listed in Appendix A, convergence tests are provided in Appendix B, and the crystal structures for all considered compounds are given in Appendix C.

The left column of Fig. 1 shows the crystal structure of $\mathrm{FeH}_{3}$, which is $P m \overline{3} m$ [24]. In Fig. 1(c), where the $\mathrm{H}-\mathrm{H}$ bonds are not shown, Fe (red) sits on a simple cubic lattice, surrounded by $12 \mathrm{H}$ nearest neighbors (blue, bond length $\simeq 1.7 \AA$ ) and by the six nearest $\mathrm{Fe}$ atoms, a factor of $\sqrt{2}$ farther away than the $\mathrm{H}$ atoms yet closer than the bulk-iron $\mathrm{Fe}-\mathrm{Fe}$ 
(a)

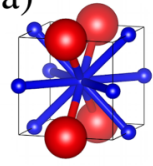

(b)

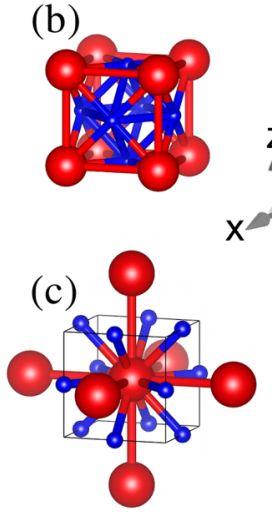

(e)

(d)

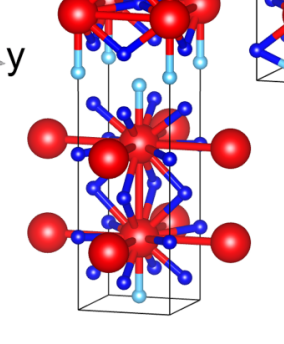

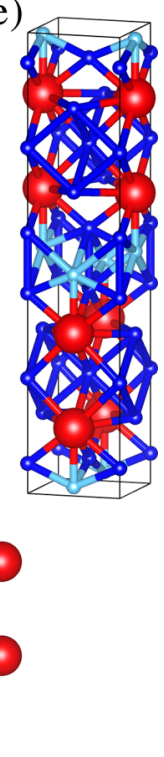

FIG. 1. $\mathrm{FeH}_{3}$ (left) and $\mathrm{FeH}_{5}$ (right) from different perspectives. $\mathrm{Fe}$ atoms are shown in red, $\mathrm{H}$ atoms are in blue and cyan, and nearestneighbor bonds are shown as bicolor sticks. The $\mathrm{FeH}_{3}$ cubic cell is chosen (a) with $\mathrm{H}$ in the middle and only its nearest-neighbor bonds shown, (b) with an fcc-like arrangement ( $\mathrm{Fe}$ in the corners, $\mathrm{H}$ in the face centers) and all the nearest-neighbor bonds shown (Fe-Fe, $\mathrm{H}-\mathrm{H}$, and $\mathrm{Fe}-\mathrm{H}$ ), and (c) with $\mathrm{Fe}$ in the middle and the $\mathrm{H}-\mathrm{H}$ nearest-neighbor bonds hidden, highlighting $12 \mathrm{H}$ nearest neighbors within the cell and six Fe nearest neighbors in the six neighboring cells. For $\mathrm{FeH}_{5}$, we adopt a tetragonal unit cell, twice as large as the primitive cell, to help visual comparison to the "parent" structure $\mathrm{FeH}_{3}$ : In cubic $\mathrm{FeH}_{3}$, the $\mathrm{Fe}$ cages are stacked along each of the three $x y z$ directions; in $\mathrm{FeH}_{5}$, instead of lining up along $z$, they are staggered to make room for the 13th (cyan) $\mathrm{H}$ atom, which saturates the vertical broken Fe-Fe bond of $\mathrm{FeH}_{3}$. The two visualizations of $\mathrm{FeH}_{5}$ correspond to (d) hiding the $\mathrm{H}-\mathrm{H}$ bonds or (e) hiding the $\mathrm{Fe}-\mathrm{Fe}$ bonds and horizontally shifting the origin along $x$ by half the horizontal lattice constant.

distance at normal pressure $(\sim 2.5 \AA)$. Figure 1 (a), where $\mathrm{H}$ is in the middle of the cube and the Fe-Fe bonds are hidden, highlights the local $\mathrm{H}$ environment, with eight $\mathrm{H}$ and four $\mathrm{Fe}$ equidistant nearest neighbors. Both Figs. 1(c) and 1(a) are obvious consequences of the fcc-like structure of $\mathrm{FeH}_{3}$ [Fig. 1(b)], i.e., a cubic cage with Fe atoms in the corners and $\mathrm{H}$ atoms in the face centers of the unit cell.

The crystal structure of $\mathrm{FeH}_{5}, \mathrm{I} / \mathrm{mmm}$, determined by Pépin et al. [19] and confirmed by evolutionary crystal structure searches [21], is shown in the right-hand column of Fig. 1, where we adopt a 4-f.u. conventional unit cell, which is twice as large as the primitive unit cell. With this choice, it can be viewed as two cubic cages of Fe, cut out of bulk $\mathrm{FeH}_{3}$ together with all their nearest-neighbor hydrogen atoms, vertically separated by a small void and displaced with respect to each other in the $x y$ plane. The empty space is occupied by additional hydrogen atoms, shown in cyan, which saturate the broken $\mathrm{Fe}-\mathrm{Fe}$ bonds above and below. Thus, compared to the cubic $\mathrm{FeH}_{3}, \mathrm{FeH}_{5}$ appears as a stack of alternating layers of saturated $\mathrm{FeH}_{3}$-like cubic cages, where each $\mathrm{Fe}$ atom binds 13 hydrogen atoms (instead of 12) and five Fe atoms (instead of six). To emphasize this point of view, Fig. 1(d) shows the Fe-H and $\mathrm{Fe}-\mathrm{Fe}$ bonds and hides the $\mathrm{H}-\mathrm{H}$ bonds, thus emphasizing the similarities between $\mathrm{FeH}_{5}$ and its precursor $\mathrm{FeH}_{3}$.

Other authors argue that all of the hydrogen atoms (i.e., both blue and cyan), which lie in the void regions between subsequent layers of Fe cubic cages, should, instead, be regarded as a dense atomic-hydrogen layer $[19,20]$; this is not just a matter of taste, as the two descriptions correspond to entirely different electronic (and hence superconducting) properties of the system. We will show in the following that such a two-dimensional (2D) metallic-hydrogen scenario, in spite of its appealing implications for high- $T_{\mathrm{c}}$ conventional superconductivity, $[2,3]$ is both geometrically and electronically unjustified.

First of all, Fig. 1(e), by displaying a different set of nearestneighbor bonds with respect to Fig. 1(d) (i.e., by hiding the $\mathrm{Fe}-\mathrm{Fe}$ bonds and displaying only the $\mathrm{H}-\mathrm{H}$ and $\mathrm{H}-\mathrm{Fe}$ bonds), reveals that the $\mathrm{H}$ network in the interstitial space between subsequent $\mathrm{Fe}$ cages (where the "additional" cyan hydrogen atoms also sit) has $\mathrm{H}-\mathrm{H}$ distances ranging from 1.3 to $1.54 \AA$, twice as large as the $\mathrm{H}_{2}$ bond length $(0.74 \AA)$ and also larger than the two $\mathrm{H}-\mathrm{H}$ distances $(0.98$ and $1.2 \AA)$ predicted for solid atomic hydrogen at $\sim 500 \mathrm{GPa}[6]$. In other words, the interstitial $\mathrm{H}$ network in $\mathrm{FeH}_{5}$ is not much denser than the $\mathrm{H}$ network within the iron cages, where $\mathrm{H}-\mathrm{H}$ distances are all equal to $\sim 1.6 \AA\left(\sim 1.65 \AA\right.$ in $\left.\mathrm{FeH}_{3}\right)$.

Second, all the Fe-H nearest-neighbor distances in $\mathrm{FeH}_{5}$ (ranging from 1.46 to $1.70 \AA$ ) are comparable to those found in $\mathrm{FeH}_{3}(1.65 \AA)$ at the same pressure, the shortest one actually corresponding to the cyan interstitial $\mathrm{H}$ atom.

Third, and most importantly, the 2D metallic-hydrogen scenario is not consistent with the electronic structure of $\mathrm{FeH}_{5}$, where, on the one hand, no bands with hydrogen-only character may be found within at least $\pm 5 \mathrm{eV}$ from the Fermi level and, on the other hand, Brillouin-zone folding effects due to the 4-f.u. unit cell are enough to explain the main features of the $\mathrm{FeH}_{5}$ band structure by tracing them back to the simpler $\mathrm{FeH}_{3}$ band structure.

This can be appreciated in Fig. 2, where the electronic band structures of $\mathrm{FeH}_{3}$ (top) and $\mathrm{FeH}_{5}$ (bottom), decorated with partial $\mathrm{Fe}$ and $\mathrm{H}$ characters, are shown.

In $\mathrm{FeH}_{3}$, the eight $\mathrm{Fe} 3 d-\mathrm{H} 1 s$ bands have a total bandwidth of $\sim 25 \mathrm{eV}$. The hydrogen $s$ states form one bonding band centered $\sim 15 \mathrm{eV}$ below $E_{F}$ and two nonbonding bands at higher energies [see Fig. 2(c)]. The five Fe bands, subdivided into $e_{g}$ and $t_{2 g}$ manifolds [see Figs. 2(a) and 2(b), respectively], fall mostly within the wide $(\sim 10 \mathrm{eV})$ gap between lower and upper $\mathrm{H}$ bands. The Fermi level of $\mathrm{FeH}_{3}$ cuts the band structure in the middle of the $t_{2 g}$ manifold, where the hybridization of $\mathrm{H} 1 s$ with $\mathrm{Fe} 3 d$ states is negligible, with a negligible $\mathrm{H}$ contribution to the density of states (DOS). The hybridization with hydrogen $1 s$ states is, instead, significant in the $e_{g}$ manifold, located $2.5 \mathrm{eV}$ above and $7.5 \mathrm{eV}$ below the Fermi level.

In $\mathrm{FeH}_{5}$, the projection on Wannier orbitals shows that the electronic bands around the Fermi level are mainly of $\mathrm{Fe} 3 d$ character in either its $e_{g}$ or $t_{2 g}$ representation [see Figs. 2(d) and 2(e), respectively]. Compared to $\mathrm{FeH}_{3}$, there are two more electrons per f.u. in $\mathrm{FeH}_{5}$, and hence, the Fermi level cuts the band structure at the top of the $t_{2 g}$ manifold in a region 
$\begin{array}{lllllllllll}\mathrm{Z} & \Gamma & \mathrm{X} & \mathrm{M} & \Gamma & \mathrm{R} & \mathrm{M} & \mathrm{DOS}\end{array}$

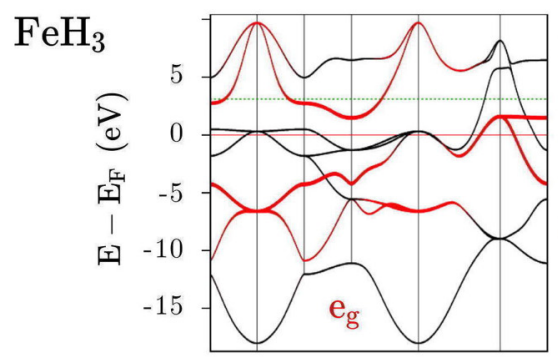

$\mathrm{FeH}_{5}$

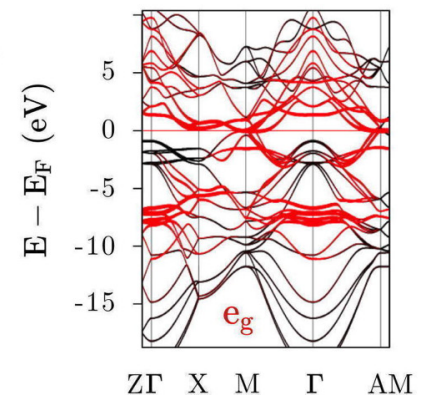

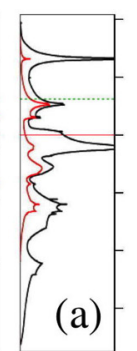

(a)

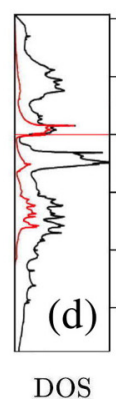

$\begin{array}{lllllllllllll}\mathrm{Z} & \Gamma & \mathrm{X} & \mathrm{M} & \Gamma & \mathrm{R} & \mathrm{M} & \mathrm{DOS}\end{array}$
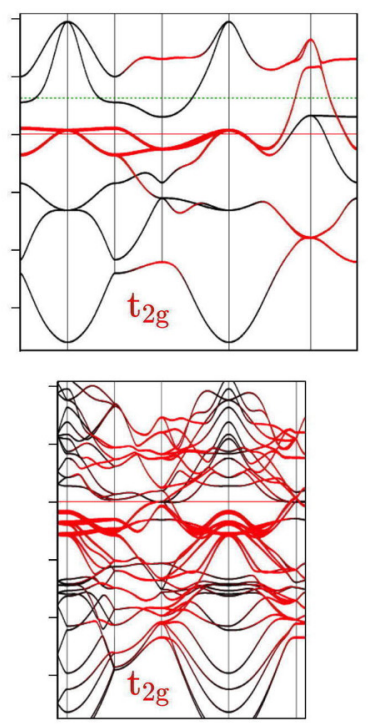

$\mathrm{Z} \Gamma \quad \mathrm{X} \quad \mathrm{M} \quad \mathrm{\Gamma} \quad \mathrm{AM}$

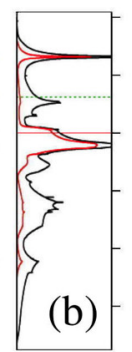

(b)

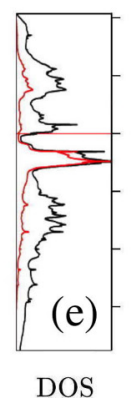

$\begin{array}{lllllllll}\mathrm{Z} & \Gamma & \mathrm{X} & \mathrm{M} & \Gamma & \mathrm{R} & \mathrm{M} & \mathrm{DOS}\end{array}$

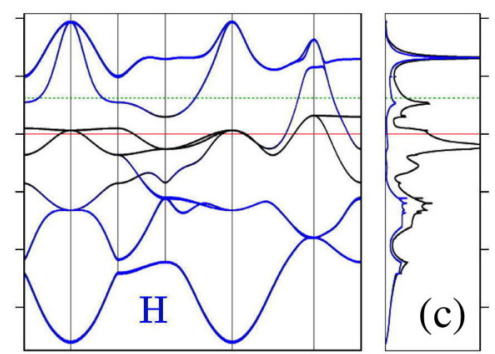

(c)
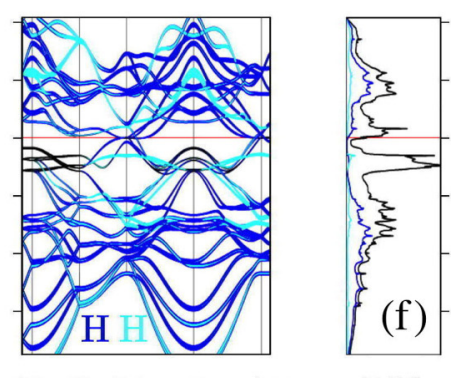

DOS

FIG. 2. Electronic band structure and DOS of $\mathrm{FeH}_{3}$ (top) and $\mathrm{FeH}_{5}$ (bottom). The energy bands (black thin lines), decorated with dots whose size is proportional to their wave-function character, are shown for (a) and (d) Fe $e_{g}$, (b) and (e) Fe $t_{2 g}$, and (c) and (f) $\mathrm{H} 1 s$ states, along with the corresponding partial DOS. Total densities of states are shown in black, red indicates $\mathrm{Fe}$ atoms, blue shows the 12 nearest-neighbor $\mathrm{H}$ of $\mathrm{Fe}$ which are present in both $\mathrm{FeH}_{3}$ and $\mathrm{FeH}_{5}$, and cyan indicates the 13th nearest neighbor of Fe which exists only in FeH (see Fig. 1). The dashed green line indicates the energy needed to dope into bands with mixed Fe $e_{g}-\mathrm{H} 1 s$ character. We use the notation for the special points of a simple tetragonal lattice for both $\mathrm{FeH}_{3}$ and $\mathrm{FeH}_{5}$ to facilitate comparison.

where the electronic DOS is extremely low and exhibits a pseudogap. Such a Fermi-level shift due to two more electrons per f.u. leaves, however, the contribution of $\mathrm{H} 1 s$ states to the DOS [see Fig. 2(f)] at the Fermi level as low as in $\mathrm{FeH}_{3}$. As a result, for both $\mathrm{FeH}_{3}$ and $\mathrm{FeH}_{5}$, the dominant contribution to the DOS at the Fermi level, and thus to superconducting pairing, comes from the Fe sublattice, not from the $\mathrm{H}$ sublattice. This in turn implies that, in the best case, these iron hydrides will behave like elemental metals $\left(T_{\mathrm{c}} \lesssim 10 \mathrm{~K}\right)$ and not like the recently discovered high-pressure superconducting hydrides $\left(T_{\mathrm{c}} \gtrsim 77 \mathrm{~K}\right)[1,7-13]$.

The above qualitative prediction is quantitatively confirmed by our $a b$ initio Migdal-Eliashberg calculations. Figure 3 shows, for $\mathrm{FeH}_{3}$ (top) and $\mathrm{FeH}_{5}$ (bottom), the phonon dispersion, the phonon DOS, and the Eliashberg $e$-ph spectral function, from whose moments we estimate a superconducting $T_{\mathrm{c}}$ using the McMillan-Allen-Dynes formula [25]:

$$
T_{\mathrm{c}}=\frac{\omega_{\log }}{1.2 k_{B}} \exp \left[-\frac{1.04(1+\lambda)}{\lambda-\mu^{*}(1+0.62 \lambda)}\right],
$$

where $\omega_{\log }$ and $\lambda$ are the logarithmic-averaged phonon frequency and the $e$-ph coupling constant, respectively, and $\mu^{*}$ is the Coulomb (Morel-Anderson) pseudopotential. Setting $\mu^{*}$ to a typical value $\left(\mu^{*}=0.16\right)$, we obtain $\omega_{\log }=65.7 \mathrm{meV}$, $\lambda=0.2, T_{\mathrm{c}}=0 \mathrm{~K}$ for $\mathrm{FeH}_{3}$ and $\omega_{\mathrm{log}}=90.5 \mathrm{meV}, \lambda=0.14$, $T_{\mathrm{c}}=0 \mathrm{~K}$ for $\mathrm{FeH}_{5}$. In other words, for both compounds the isotropic version of the Migdal-Eliashberg theory predicts no conventional superconductivity (we double-checked that this result holds even within the fully anisotropic theory) $[22,26,27]$.

Our results for $\mathrm{FeH}_{3}$ agree with those of other authors [21]. For $\mathrm{FeH}_{5}$, instead, our findings are in remarkable disagreement with two previous studies on the same compound, which both predict a substantial $T_{\mathrm{c}}$ of around $50 \mathrm{~K}[20,21]$. As detailed in Appendix B, we have tested several possible sources of discrepancy, but all calculations with physically justifiable

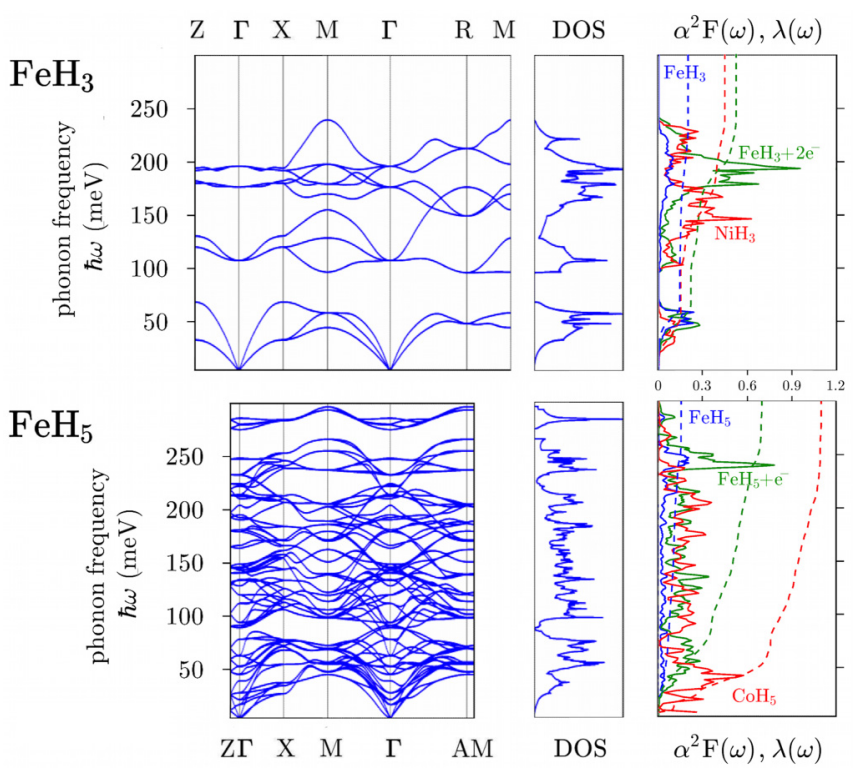

FIG. 3. Phonon dispersion (left), phonon DOS (middle), and Eliashberg function $\alpha^{2} F(\omega)$ [right, Eq. (2)]. The top panels refer to $\mathrm{FeH}_{3}$ (blue curves), where Eq. (2) was also evaluated for two-electrondoped $\mathrm{FeH}_{3}$ (green) and for $\mathrm{NiH}_{3}$ (red). The dashed curves indicate the frequency-dependent coupling constant $\lambda(\omega)$. The bottom panels refer to $\mathrm{FeH}_{5}$ (blue curves), where Eq. (2) was also evaluated for one-electron-doped $\mathrm{FeH}_{5}$ (green) and unstable $\mathrm{CoH}_{5}$ (red; see text). 
parameters invariably yielded a vanishing $T_{\mathrm{c}}$ for $\mathrm{FeH}_{5}$. Our study on the dependence of $T_{\mathrm{c}}$ on doping in the rigid-band approximation corroborates these findings.

Before presenting this additional study, we want to explain how the superconducting trends in $\mathrm{FeH}_{3}$ and $\mathrm{FeH}_{5}$ can be understood on qualitative grounds. The main ingredient of the conventional theory of superconductivity is the Eliashberg function

$$
\alpha^{2} F(\omega)=\frac{1}{N\left(E_{F}\right)} \sum_{\mathbf{k q}, v}\left|g_{\mathbf{k}, \mathbf{k}+\mathbf{q}, v}\right|^{2} \delta\left(\epsilon_{\mathbf{k}}\right) \delta\left(\epsilon_{\mathbf{k}+\mathbf{q}}\right) \delta\left(\omega-\omega_{\mathbf{q}, v}\right),
$$

from which the parameters of the McMillan-Allen-Dynes formula (1) are obtained as $\lambda=2 \int \frac{d \omega}{\omega} \alpha^{2} F(\omega), \omega_{\log }=$ $\exp \left[\frac{2}{\lambda} \int \frac{d \omega}{\omega} \alpha^{2} F(\omega) \ln (\omega)\right]$. In Eq. (2), $N\left(E_{F}\right)$ is the DOS at the Fermi level, $\omega_{\mathbf{q}, v}$ is the phonon frequency of mode $v$ and wave vector $\mathbf{q}$, and $\left|g_{\mathbf{k}, \mathbf{k}+\mathbf{q}, v}\right|$ is the $e$-ph matrix element between two electronic states of wave vectors $\mathbf{k}$ and $\mathbf{k}+\mathbf{q}$ at the Fermi level [28]. The double-delta function $\delta\left(\varepsilon_{\mathbf{k}}^{n}\right) \delta\left(\varepsilon_{\mathbf{k}+\mathbf{q}}^{m}\right)$ restricts the sum of $e$-ph matrix elements to electronic states at the Fermi level.

High- $T_{\mathrm{c}}$ conventional superconductors are compounds where the double- $\delta$ function in Eq. (2) selects electronic states with a large $\left|g_{\mathbf{k}, \mathbf{k}+\mathbf{q}, v}\right|$, i.e., electronic states which are strongly modified by the ionic motion. A real-space-resolved electronic DOS, the so-called local density of states (LDOS), defined as

$$
N(E, \mathbf{r})=\frac{1}{(2 \pi)^{3}} \sum_{n} \int d^{3} k \delta\left(E-\varepsilon_{\mathbf{k}}^{n}\right)\left|\psi_{n \mathbf{k}}(\mathbf{r})\right|^{2}
$$

and evaluated at the Fermi level, provides visual intuition of why the $e$-ph coupling is large or small in a given compound. Also, when evaluated at other selected energies, it may tell something about the $e$-ph coupling of a particular band (or band manifold).

To this end we present in Fig. 4, for $\mathrm{FeH}_{3}$ [Fig. 4(a)] and $\mathrm{FeH}_{5}$ [Fig. 4(d)], the LDOS at the Fermi level $N\left(E_{F}, \mathbf{r}\right)$ along the (100) lattice plane, which cuts through $\mathrm{Fe}$ and $\mathrm{H}$ atoms in both $\mathrm{FeH}_{3}$ and $\mathrm{FeH}_{5}$ [see Figs. 1(b) and 1(d)]. A red arrow connects each of these two panels with the red horizontal line highlighting the Fermi level of the corresponding band structure.

Since, as already mentioned when discussing Fig. 2, the Fermi level falls within the Fe $t_{2 g}$ bands (in $\mathrm{FeH}_{3}$ among them, in $\mathrm{FeH}_{5}$ at their top) and since the $\mathrm{H}$ contribution to the electronic DOS at $E_{F}$ is negligible in both compounds, it is readily understood that the LDOS pattern, although qualitatively different (since $E_{F}$ does not cut the $t_{2 g}$ bands in the same place), appears intense around the $\mathrm{Fe}$ atoms in both compounds and either undetectable $\left[\mathrm{FeH}_{3}\right.$, Fig. 4(a)] or barely visible $\left[\mathrm{FeH}_{5}\right.$, Fig. 4(d)] around the $\mathrm{H}$ atoms and in the interstitial regions. From these Fe-dominated bands we expect almost no coupling to the $\mathrm{H}$ motion and an $e$-ph coupling to the Fe motion as low as in bulk iron (not a superconductor). Indeed, in both in the top $\left(\mathrm{FeH}_{3}\right)$ and bottom $\left(\mathrm{FeH}_{5}\right)$ panels of Fig. 3, the corresponding Eliashberg functions $\alpha^{2} F(\omega)$ show a very low average $e$-ph coupling, uniformly spread over Fe and $\mathrm{H}$ modes.

Not all of the electronic states in these transition-metal polyhydrides, however, have such a poor intrinsic $e$-ph coupling. If, by doping, one added more electrons to the system, one could completely fill the $t_{2 g}$ bands, and the Fermi level would eventually reach a band which has a mixed Fe $e_{g}-\mathrm{H} 1 s$ character. In $\mathrm{FeH}_{3}$ such an $\mathrm{Fe}-\mathrm{H}$ hybrid band, highlighted by a horizontal dashed green line in Fig. 4 (and also in Fig. 2), starts at $\sim 2 \mathrm{eV}$ above the Fermi level along the $X-M$ segments. In $\mathrm{FeH}_{5}$, due to the different electron count and to Brillouin-zone folding effects, the same Fe-H hybrid band can be found just $\sim 0.5 \mathrm{eV}$ above $E_{F}$ (other replicas appear above it as well).

In Fig. 4, a green arrow connects the mean energy of each of these bands to the corresponding plot of the LDOS in the (100) lattice plane, shown in the Figs. 4(b) and 4(c) for $\mathrm{FeH}_{3}$ and $\mathrm{FeH}_{5}$, respectively. For both compounds, the LDOS displays the same $\left(e_{g}\right)$ symmetry around the Fe atom at this energy, and unlike the bands at the Fermi level, it also displays a considerable weight (bright yellow spots) around the $\mathrm{H}$ atoms. For electronic states with such a real-space distribution, it is reasonable to expect a sizable $e$-ph coupling since they are likely to be affected by both $\mathrm{H}$ and $\mathrm{Fe}$ vibrations. The real-space distribution itself suggests, however, that the $e$-ph coupling will be moderate because of the lack of directional bonds between $\mathrm{Fe}$ and $\mathrm{H}$. As a matter of fact, the LDOS at the Fermi level in Fig. 4 shows no evidence of any directional bonds at all, as if all sticks between pairs of atoms had been removed in Fig. 1.

A rough quantitative estimate of the $e$-ph coupling of the aforementioned $\mathrm{Fe}-\mathrm{H}$ hybrid electronic states may be given by simply recomputing the Eliashberg function (2) after a rigid shift of the Fermi level into that energy band. Figure 5 shows as red squares the calculated $e$-ph and superconducting properties (i.e., $\lambda$ and $T_{\mathrm{c}}$ ) as a function of electron count for our two compounds. We see that the threshold to bring the Fermi level into the $\mathrm{Fe}-\mathrm{H}$ hybrid band is between 13 and $14 e^{-} /$f.u., which implies at least two additional $e^{-} /$f.u. in $\mathrm{FeH}_{3}$ but only $\sim 0.25 e^{-} /$f.u. in $\mathrm{FeH}_{5}$. We also see that, in agreement with our expectations, the $e$-ph coupling experiences an abrupt increase as soon as the respective doping thresholds are exceeded.

This numerical rigid-band experiment is instructive for understanding the key features of the $\mathrm{FeH}_{3} / \mathrm{FeH}_{5}$ electronic structure and $e$-ph coupling but is not a practical way to improve the superconducting properties of real materials. This can, instead, be achieved by chemical means, i.e., by replacing Fe with other transition elements with more $d$ electrons. To this end, we have studied the $e$-ph coupling properties of $X \mathrm{H}_{3}$ compounds in the $P m \overline{3} m$ structure, with $X=\mathrm{Co}$ $\left(+1 e^{-}\right), \mathrm{Ni}\left(+2 e^{-}\right)$, and $\mathrm{Cu}\left(+3 e^{-}\right)$. For $X=\mathrm{Co}$ and $\mathrm{Ni}$ we obtain a dynamically stable $P m \overline{3} m$ structure, and evolutionary structure searches consistently converge to such a $P m \overline{3} m$ structure at $150 \mathrm{GPa}$. $\mathrm{Cu}$, on the other hand, is dynamically unstable, and in this case analogous searches run into highly distorted structures.

The results of these simulations are shown as blue dots in Fig. 5, where our previous rigid-band-doping estimates are marked as red squares. Our results for actual compounds follow the rigid-band-doping trends reasonably, confirming our understanding of the $e$-ph mechanism in this class of compounds. In particular, we see that the addition of electrons to $\mathrm{FeH}_{3}$ (by replacing $\mathrm{Fe}$ with $\mathrm{Ni}$ ) is a more effective way to increase $\lambda$ (bottom panel) than the addition of $\mathrm{H}$ atoms, as in $\mathrm{FeH}_{5}$. In connection with these calculations, we also report the Eliashberg functions for $\mathrm{FeH}_{3}, \mathrm{FeH}_{3}+2 e^{-}$, and $\mathrm{NiH}_{3}$ in the top right panel of Fig. 3, which corroborates our findings: 


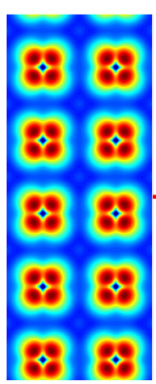

(a)

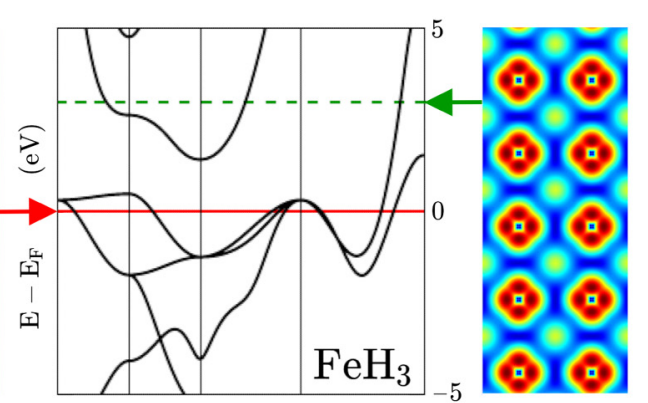

$\begin{array}{lllll}\Gamma & \mathrm{X} & \mathrm{M} & \Gamma & \mathrm{R}\end{array}$ (b)

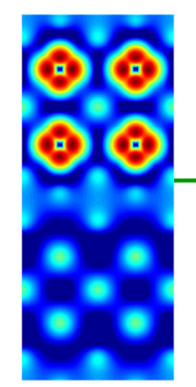

(c)

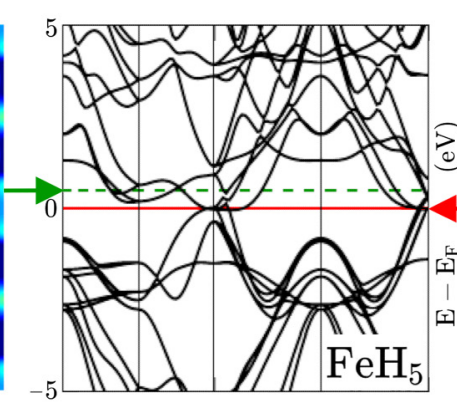

$\begin{array}{lllllllll}\Gamma & \mathrm{X} & \mathrm{M} & \Gamma & \mathrm{A}\end{array}$

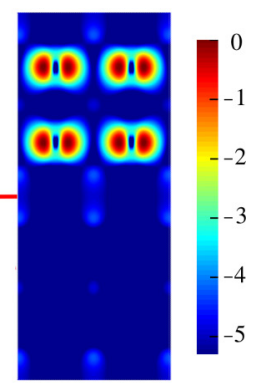

(d)

FIG. 4. Low-energy band structure and LDOS [Eq. (3)] for (a) and (b) $\mathrm{FeH}_{3}$ and (c) and (d) $\mathrm{FeH}_{5}$ along a (100) plane, which cuts through Fe and $\mathrm{H}$ atoms in both compounds (see Fig. 1). In (a) and (d) the LDOS is displayed at the Fermi level of the corresponding compounds (red arrows); in (b) and (c) the LDOS is displayed at the energy of an empty Fe-H hybrid band above the respective Fermi levels (green arrows; see text), corresponding to an electron count of $\mathrm{FeH}_{3}+2 e^{-}$and $\mathrm{FeH}_{5}+0.5 e^{-}$in Fig. 5. The color scale used corresponds to $\ln [\mathrm{LDOS} / \mathrm{max}(\mathrm{LDOS})]$ to improve visibility.

Moving the Fermi level more and more into the Fe-H hybrid band causes a progressive increase of the $e$-ph coupling at high energies, where $\mathrm{Fe}-\mathrm{H}$ modes are concentrated. Indeed, as soon as two electrons are added to the system $\left(\mathrm{FeH}_{3}+2 e^{-}\right.$, green) a large peak centered at $\sim 180 \mathrm{meV}$ appears, which is absent in $\mathrm{FeH}_{3}$ (blue). This peak shifts to lower energies $(\sim 150 \mathrm{meV})$ in $\mathrm{NiH}_{3}$ (red) because the corresponding phonon frequencies are shifted down by electronic screening effects (see Appendix D). In the top panel of Fig. 5 we see, however, that the actual substitution of $\mathrm{Fe}$ with $\mathrm{Ni}$ is not sufficient to yield appreciable superconductivity. In spite of the larger $\lambda$ in $\mathrm{NiH}_{3}$,

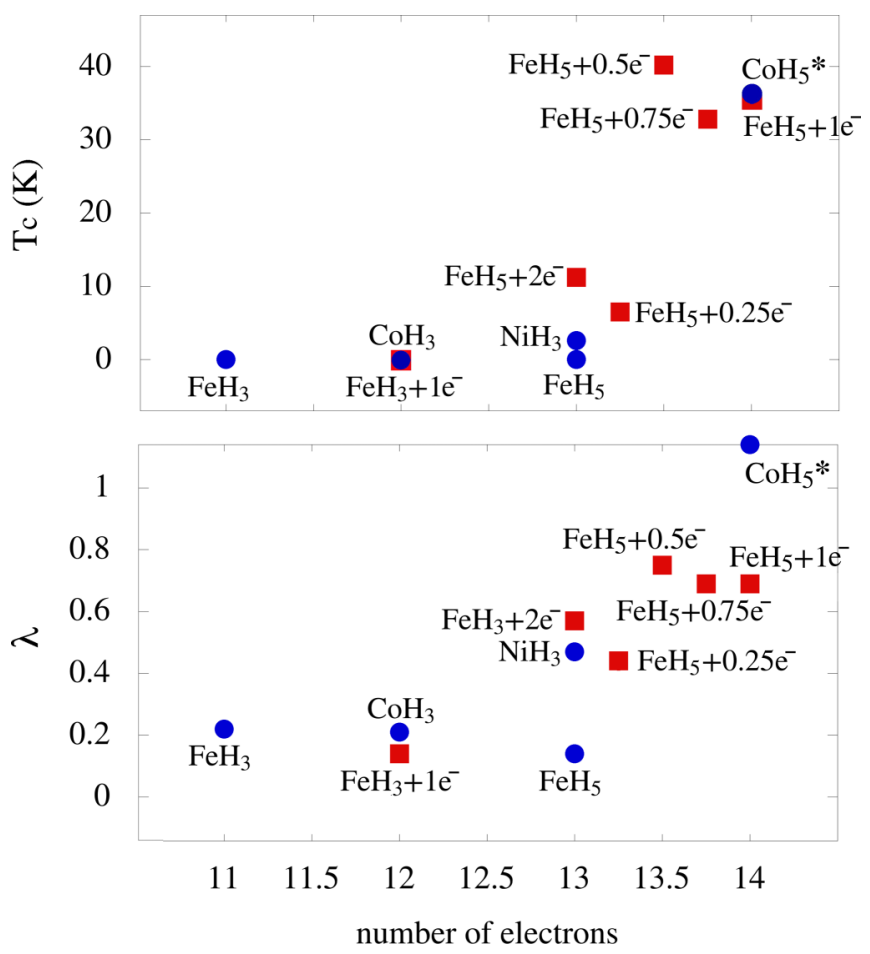

FIG. 5. Critical temperature $T_{\mathrm{c}}$ (top panel) and $e$-ph coupling constant $\lambda$ (bottom panel) for a number of transition-metal polyhydrides. Blue dots indicate first-principles results for stable $\mathrm{FeH}_{3}, \mathrm{CoH}_{3}, \mathrm{NiH}_{3}$, and $\mathrm{FeH}_{5}$ and for unstable $\mathrm{CoH}_{5}^{*}$; red squares refer to rigid-band doping (see text). the renormalization of phonon frequencies and matrix elements due to the electronic screening brings $T_{\mathrm{c}}$ down to $\sim 3 \mathrm{~K}$, compared to the $11 \mathrm{~K}$ of the corresponding rigid-band result. Adding more electrons amplifies such effects, making $\mathrm{CuH}_{3}$ (not shown in Fig. 5) dynamically unstable in this structure, as mentioned previously.

For similar reasons $\mathrm{CoH}_{5}$, which, according to our rigidband prediction, should be an $\sim 35 \mathrm{~K}$ superconductor (in the same $I 4 / \mathrm{mmm}$ structure as $\mathrm{FeH}_{5}$ ), turns out to be dynamically unstable in the harmonic approximation, with imaginary phonon frequencies over large portions of the Brillouin zone (see Appendix D), indicating that $\mathrm{CoH}_{5}$ will, most likely, not form in this structure at this pressure. Nevertheless, if we compute its $e$-ph coupling properties by integrating the Eliashberg function over the real portion of the phonon spectrum, we obtain a large value for the coupling $\lambda$ and a $T_{\mathrm{c}}$ considerably larger than that of $\mathrm{FeH}_{5}$. The bottom panels of Fig. 3 show a comparison of $\alpha^{2} F(\omega)$ of $\mathrm{CoH}_{5}$ with the corresponding rigid-band result; here, too, we observe increased coupling at large frequencies, but in $\mathrm{CoH}_{5}$ part of the corresponding spectrum is shifted to negative $\omega^{2}$ (imaginary frequencies), which we exclude from the integral. This explains why the total $e$-ph coupling of $\mathrm{CoH}_{5}$ is lower than that of the rigid-band calculation. We did not pursue the $\mathrm{CoH}_{5}$ experiment any further for two reasons: (i) the presence of the $\mathrm{Fe}-\mathrm{H}$ hybrid band depends crucially on the crystal structure, and the dynamical instability we found indicates an important lattice distortion. (ii) Even if, in principle, anharmonic effects, which can be sizable in high-pressure hydrides [29], could stabilize the $I 4 / \mathrm{mmm}$ structure, in practice, a recent study [30] found that the $\mathrm{CoH}_{5}$ stoichiometry is not thermodynamically stable up to $300 \mathrm{GPa}$; that is, it would be extremely difficult to stabilize it in other structures as well. What we found so far is enough to strongly suggest that a sizable $T_{\mathrm{c}}$ cannot be obtained by simple chemical means in $\mathrm{XH}_{5}$ compounds.

\section{CONCLUSIONS}

In summary, we have computed from first principles the electronic, structural, and superconducting properties of the new iron hydride $\mathrm{FeH}_{5}$, recently synthesized by Pépin $e t$ al. [19]. At variance with two previous studies $[20,21]$ we found 

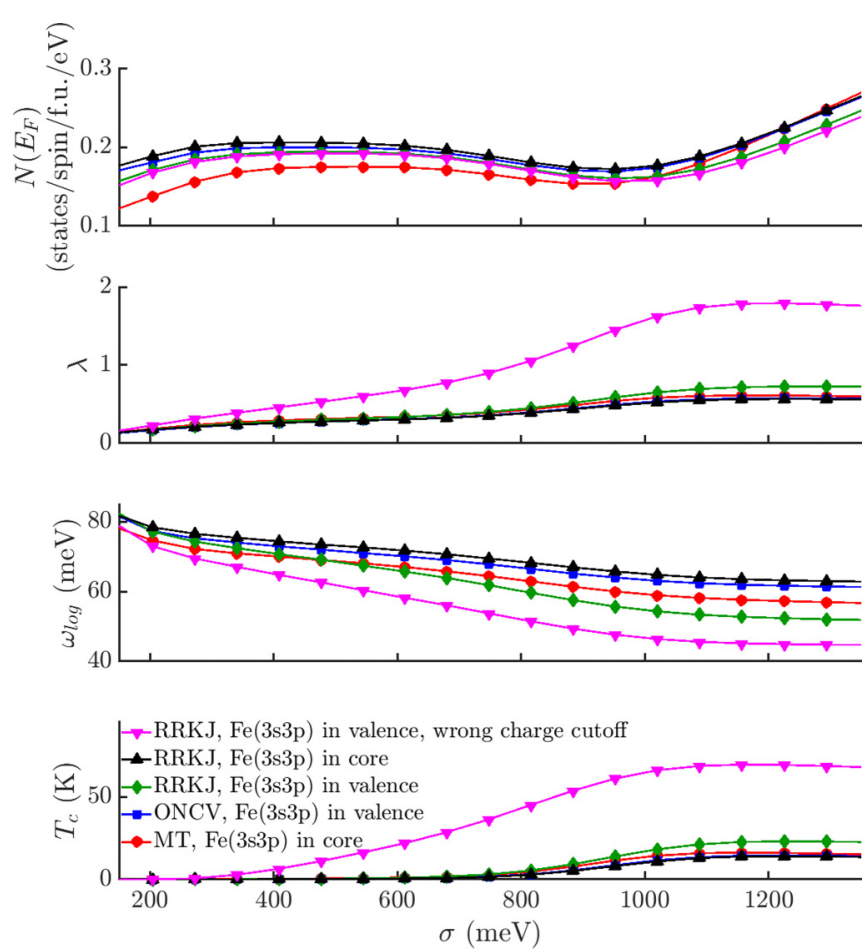

FIG. 6. $N\left(E_{F}\right)$ (first panel), $\lambda$ (second panel), $\omega_{\log }$ (third panel), and $T_{\mathrm{c}}$ (fourth panel) of $\mathrm{FeH}_{5}$ as a function of electronic smearing $\sigma$ and the pseudopotential $[31,36,37]$.

that $\mathrm{FeH}_{5}$ is not superconducting and showed that this had to be expected since its electronic states at the Fermi level, dominated by $d$-metal bonding, have an intrinsically low $e$-ph coupling. Moreover, the only band with appreciable Fe-H hybridization and $e$-ph coupling lies above the Fermi level and is thus inaccessible to superconductivity. We exploited doping to shift the Fermi level into this Fe-H hybrid band and, indeed, obtained a higher $T_{\mathrm{c}}$ but found that, even so, $T_{\mathrm{c}}$ would hardly reach $40 \mathrm{~K}$. Moreover, even this value is practically impossible to achieve, as the required doping levels push these compounds beyond their structural stability limits. The picture emerging from our analysis strongly contradicts the notion, proposed by other authors $[19,20]$, of a layer of dense metallic hydrogen dominating the superconducting properties of $\mathrm{FeH}_{5}$. Our results do not translate into the identification of new candidates for high- $T_{\mathrm{c}}$ superconductivity in the $\mathrm{FeH}_{x}$ family; on the contrary, they rule out this class of compounds from the list of potential high- $T_{\mathrm{c}}$, high-pressure superconductors, shedding new light on the mechanisms leading to high $T_{\mathrm{c}}$ in high-pressure hydrides.

\section{ACKNOWLEDGMENTS}

This work was supported by Austrian Science Fund (FWF) Projects No. J 3806-N36 and P 30269-N36, the dCluster of the Graz University of Technology, and the VSC3 of the Vienna University of Technology. L.B. and G.B.B. acknowledge support from Fondo Ateneo-Sapienza 2017.
TABLE I. Crystal structures of the compounds mentioned in the main text. The atomic positions are given in crystal coordinates, along with their Wyckoff positions in square brackets.

\begin{tabular}{cccccc}
\hline \hline & \multirow{2}{*}{ Space } & Lattice & \multicolumn{3}{c}{ Atomic positions } \\
\cline { 4 - 6 } & & group & parameters (A) & Atom Wyckoff & Crystal \\
\hline $\mathrm{FeH}_{3}$ & $P m \overline{3} m$ & $a=b=c=2.33$ & $\mathrm{Fe}$ & {$[1 \mathrm{a}]$} & $(0.00,0.00,0.00)$ \\
& & & $\mathrm{H}$ & {$[3 \mathrm{c}]$} & $(0.00,0.50,0.50)$ \\
$\mathrm{CoH}_{3}$ & $P m \overline{3} m$ & $a=b=c=2.33$ & $\mathrm{Co}$ & {$[1 \mathrm{a}]$} & $(0.00,0.00,0.00)$ \\
& & & $\mathrm{H}$ & {$[3 \mathrm{c}]$} & $(0.00,0.50,0.50)$ \\
$\mathrm{NiH}_{3}$ & $P m \overline{3} m$ & $a=b=c=2.35$ & $\mathrm{Ni}$ & {$[1 \mathrm{a}]$} & $(0.00,0.00,0.00)$ \\
& & & $\mathrm{H}$ & {$[3 \mathrm{c}]$} & $(0.00,0.50,0.50)$ \\
$\mathrm{FeH}_{5}$ & $I 4 / m m m$ & $a=b=2.39$ & $\mathrm{Fe}$ & {$[4 \mathrm{e}]$} & $(0.00,0.00,0.10)$ \\
& & $c=11.50$ & $\mathrm{H}$ & {$[8 \mathrm{~g}]$} & $(0.00,0.50,0.32)$ \\
& & & $\mathrm{H}$ & {$[4 \mathrm{e}]$} & $(0.00,0.00,0.59)$ \\
& & & $\mathrm{H}$ & {$[4 \mathrm{c}]$} & $(0.00,0.50,0.00)$ \\
& & & $\mathrm{H}$ & {$[4 \mathrm{e}]$} & $(0.00,0.00,0.77)$ \\
$\mathrm{CoH}_{5} I 4 / m m m$ & $a=b=2.40$ & $\mathrm{Co}$ & {$[4 \mathrm{e}]$} & $(0.00,0.00,0.10)$ \\
& & $c=11.39$ & $\mathrm{H}$ & {$[8 \mathrm{~g}]$} & $(0.00,0.50,0.32)$ \\
& & & $\mathrm{H}$ & {$[4 \mathrm{e}]$} & $(0.00,0.00,0.59)$ \\
& & & $\mathrm{H}$ & {$[4 \mathrm{c}]$} & $(0.00,0.50,0.00)$ \\
& & & $\mathrm{H}$ & {$[4 \mathrm{e}]$} & $(0.00,0.00,0.77)$ \\
& & & & &
\end{tabular}

\section{APPENDIX A: COMPUTATIONAL DETAILS}

Our calculations were carried out using optimized normconserving Vanderbilt pseudopotentials [31,32] within the Perdew-Burke-Ernzerhof functional [33] that include the semicore electrons of Fe. We employed the QUANTUM ESPRESSO package [34] for the electronic structure and lattice dynamics, the EPW code [22] for the $e$-ph interaction and the superconducting properties, and the WANNIER90 code [35] for generating maximally localized Wannier functions. The vibrational properties were obtained using density functional perturbation theory. In all calculations for the density functional theory ground state we used a kinetic cutoff for the plane waves of $65 \mathrm{Ry}$ and a Gaussian smearing $\sigma$ of $0.01 \mathrm{Ry}$. For $\mathrm{FeH}_{3}$, we sampled the Brillouin zone for the electronic properties using a $24 \times 24 \times 24$ grid and an $8 \times 8 \times 8$ grid for the vibrational properties. Within EPW, all quantities were interpolated onto $30 \times 30 \times 30$ grids using eight Wannier functions. For $\mathrm{FeH}_{5}$ in the 4-f.u. cell (2-f.u. cell), we sampled the Brillouin zone for the electronic properties using a $24 \times 24 \times 6(24 \times 24 \times 12)$ grid and an $8 \times 8 \times 2(6 \times 6 \times 3)$ for the vibrational properties. Within EPW, all quantities were interpolated onto $32 \times 32 \times 8$ $(30 \times 30 \times 10)$ grids using $40(20)$ Wannier functions. In all calculations for superconducting properties in EPW, the Matsubara frequency cutoff was set to $1 \mathrm{eV}$, and the Dirac $\delta$ were replaced by Lorentzians with a width of $25 \mathrm{meV}$ (electrons) and $0.05 \mathrm{meV}$ (phonons).

\section{APPENDIX B: CONVERGENCE TESTS}

As our results for the superconducting parameters of $\mathrm{FeH}_{5}$ are in variance with Refs. [20,21], we performed several tests to check our results. First, since the data presented in the main text were obtained with the EPW code [22], we checked that a calculation strictly within the QUANTUM ESPRESSO package 

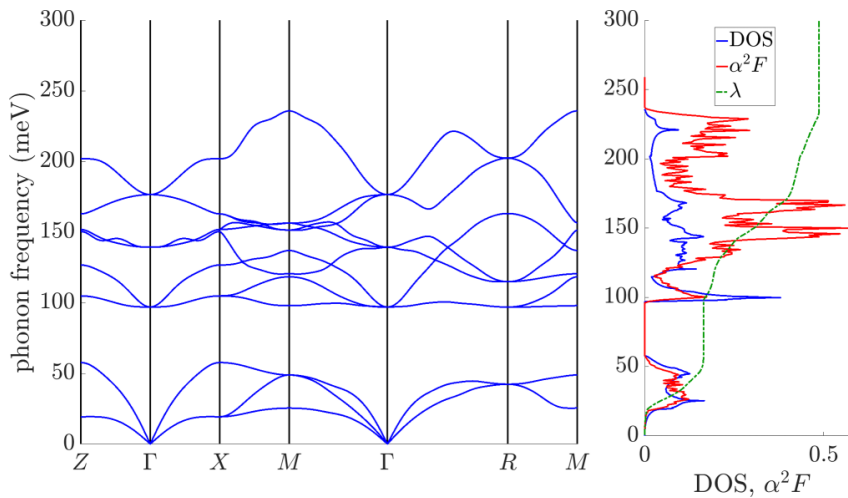

FIG. 7. Phonon dispersion, phonon DOS, $\alpha^{2} F$, and integrated $\lambda$ for $\mathrm{NiH}_{3}$.

[34], as performed in Refs. [20,21], leads to the same results. In this case, we get $\lambda=0.22, \omega_{\log }=87.6 \mathrm{meV}$, and $T_{\mathrm{c}}=0 \mathrm{~K}$, in good agreement with the results from the more elaborate EPW calculation $\left(\lambda=0.14, \omega_{\mathrm{log}}=90.5 \mathrm{meV}, T_{c}=0 \mathrm{~K}\right)$. The difference between the results can be explained by taking into account that in EPW, we used much denser Brillouin-zone grids for the integration of both electronic and vibrational properties, which allowed us to use much smaller smearing parameters ( $25 \mathrm{meV}$ for electrons and $0.05 \mathrm{meV}$ for phonons). Having established the reliability of the QUANTUM ESPRESSO results, all further convergence tests are performed using only this code, as employed in Refs. [20,21].

In Fig. 6, we show the DOS at the Fermi level $N\left(E_{F}\right)$ (first panel), electron-phonon coupling $\lambda$ (second panel), logarithmic-averaged phonon frequency $\omega_{\log }$ (third panel), and superconducting critical temperature $T_{\mathrm{c}}$ (fourth panel) of $\mathrm{FeH}_{5}$ as a function of electronic smearing $\sigma$ and pseudopotential (PP). The tested PPs include the norm-conserving MartinsTroullier [36] (MT) type, the optimized norm-conserving Vanderbilt [31] (ONCV) type, and the ultrasoft Rappe-RabeKaxiras-Joannopoulos [37] (RRKJ) type. For these convergence calculations, we used the 4-f.u. cell, a Brillouin-zone grid of $16 \times 16 \times 4$ for the electronic properties, and a $4 \times$ $4 \times 1$ grid for the vibrational properties to match the grids of Refs. [20,21]. We used a kinetic-energy cutoff of 65 Ry for the plane waves and $\mu^{*}=0.1$ to calculate $T_{\mathrm{c}}$.

In the case of the norm-conserving PPs, the MT type (red lines with circles) considers the $\mathrm{Fe} 3 s$ and $3 p$ states to be in the core, while the ONCV type (blue lines with squares) includes these states in the valence. Despite the differences, however, both these PPs lead to very similar results with respect to the superconducting properties, as is apparent from Fig. 6. The same holds true when comparing the results for the ultrasoft RRKJ with the Fe $3 s$ and $3 p$ states in the core and in the valence (black line with upwards-pointing triangles and green line with diamonds, respectively), where only little differences are appreciable. As a function of electronic smearing $\sigma$, we find

[1] A. P. Drozdov, M. I. Eremets, I. Troyan, V. Ksenofontov, and S. I. Shylin, Nature (London) 525, 73 (2015).

[2] N. W. Ashcroft, Phys. Rev. Lett. 21, 1748 (1968).

[3] N. W. Ashcroft, Phys. Rev. Lett. 92, 187002 (2004).
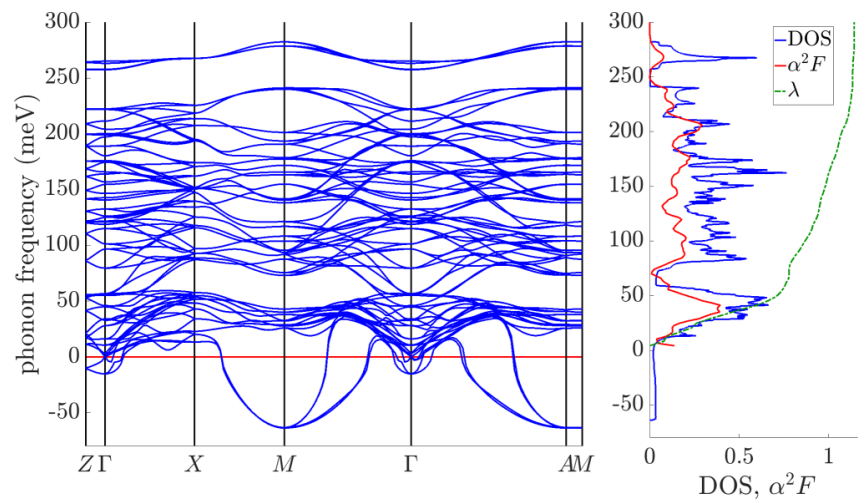

FIG. 8. Phonon dispersion, phonon DOS, $\alpha^{2} F$, and integrated $\lambda$ for $\mathrm{CoH}_{5}$.

that all previously discussed PPs yield very similar results: For $\sigma<500 \mathrm{meV}$ we get an $e$-ph coupling $\lambda<0.3$ and values for $T_{\mathrm{c}}<1 \mathrm{~K}$. Even for extremely large $\sigma>1000 \mathrm{meV}$, which is definitely too large to obtain reasonable results, the values for $\lambda$ are below 0.75 and for $T_{\mathrm{c}}<23 \mathrm{~K}$. We also find that the effects of using a larger $8 \times 8 \times 1$ Brillouin-zone grid for the vibrational properties on the superconducting properties are small. For example, we find for the ONCV case that for a smearing of $270 \mathrm{meV}, \lambda$ increases from 0.21 to 0.26 , and $T_{\mathrm{c}}$ increases from 0 to $1 \mathrm{~K}$. These differences, which keep decreasing with increasing $\sigma$, can be considered negligible for our current discussion.

However, when using ultrasoft pseudopotentials, one needs to choose a considerably larger energy cutoff for the electron density than with norm-conserving PPs, usually in the range of around 12 times the kinetic-energy cutoff. The previously discussed green and black curves, for example, were calculated using a density cutoff of $800 \mathrm{meV}$. The effect of using a too small electron density cutoff can be seen in the magenta line with downwards-pointing triangles in Fig. 6, where we used a too small electron density cutoff of only $260 \mathrm{meV}$, i.e., only 4 times the kinetic-energy cutoff. Only in this case and using very large values for $\sigma$ were we able to reproduce the results of Refs. [20,21], i.e., $\lambda>0.9$ and $T_{\mathrm{c}}>40 \mathrm{~K}$.

\section{APPENDIX C: CRYSTAL STRUCTURES}

In Table I, we report the crystal structures of all compounds mentioned in the main text.

\section{APPENDIX D: VIBRATIONAL AND ELECTRON-PHONON PROPERTIES OF $\mathrm{NiH}_{3}$ AND $\mathrm{CoH}_{5}$}

Figures 7 and 8 show the phonon dispersion, phonon DOS, $\alpha^{2} F$, and integrated $\lambda$ for $\mathrm{NiH}_{3}$ and $\mathrm{CoH}_{5}$, respectively. Due to the computational expense, the vibrational properties for $\mathrm{CoH}_{5}$ have been calculated only with QUANTUM ESPRESSO on a $4 \times 4 \times 1$ Brillouin-zone grid in the 4 -f.u. cell.

[4] P. Cudazzo, G. Profeta, A. Sanna, A. Floris, A. Continenza, S. Massidda, and E. K. U. Gross, Phys. Rev. Lett. 100, 257001 (2008).

[5] J. M. McMahon and D. M. Ceperley, Phys. Rev. B 84, 144515 (2011). 
[6] M. Borinaga, I. Errea, M. Calandra, F. Mauri, and A. Bergara, Phys. Rev. B 93, 174308 (2016).

[7] J. A. Flores-Livas, A. Sanna, and E. K. Gross, Eur. Phys. J. B 89, 63 (2016).

[8] D. Duan, Y. Liu, F. Tian, D. Li, X. Huang, Z. Zhao, H. Yu, B. Liu, W. Tian, and T. Cui, Sci. Rep. 4, 6968 (2014).

[9] I. Errea, M. Calandra, C. J. Pickard, J. Nelson, R. J. Needs, Y. Li, H. Liu, Y. Zhang, Y. Ma, and F. Mauri, Phys. Rev. Lett. 114, 157004 (2015).

[10] A. Drozdov, M. Eremets, and I. Troyan, arXiv:1508.06224.

[11] J. A. Flores-Livas, M. Amsler, C. Heil, A. Sanna, L. Boeri, G. Profeta, C. Wolverton, S. Goedecker, and E. K. U. Gross, Phys. Rev. B 93, 020508 (2016).

[12] A. Shamp, T. Terpstra, T. Bi, Z. Falls, P. Avery, and E. Zurek, J. Am. Chem. Soc 138, 1884 (2016).

[13] Y. Fu, X. Du, L. Zhang, F. Peng, M. Zhang, C. J. Pickard, R. J. Needs, D. J. Singh, W. Zheng, and Y. Ma, Chem. Mater 28, 1746 (2016).

[14] H. Wang, J. S. Tse, K. Tanaka, T. Iitaka, and Y. Ma, Proc. Natl. Acad. Sci. U.S.A 109, 6463 (2012).

[15] I. A. Kruglov, A. G. Kvashnin, A. F. Goncharov, A. R. Oganov, S. Lobanov, N. Holtgrewe, S. Jiang, V. Prakapenka, E. Greenberg, and A. V. Yanilkin, arXiv:1708.05251.

[16] H. Liu, I. I. Naumov, R. Hoffmann, N. W. Ashcroft, and R. J. Hemley, Proc. Natl. Acad. Sci. U.S.A. 114, 6990 (2017).

[17] C. Heil and L. Boeri, Phys. Rev. B 92, 060508 (2015).

[18] J. A. Flores-Livas, A. Sanna, M. Graužinyté, A. Davydov, S. Goedecker, and M. A. L. Marques, Sci. Rep. 7, 6825 (2017).

[19] C. M. Pépin, G. Geneste, A. Dewaele, M. Mezouar, and P. Loubeyre, Science 357, 382 (2017).

[20] A. Majumdar, J. S. Tse, M. Wu, and Y. Yao, Phys. Rev. B 96, 201107 (2017).

[21] A. G. Kvashnin, I. A. Kruglov, D. V. Semenok, and A. R. Oganov, J. Phys. Chem. C 122, 4731 (2018).
[22] S. Poncé, E. R. Margine, C. Verdi, and F. Giustino, Comput. Phys. Commun. 209, 116 (2016).

[23] A. O. Lyakhov, A. R. Oganov, T. H. Stokes, and Q. Zhu, Comput. Phys. Commun. 184, 1172 (2013).

[24] C. M. Pépin, A. Dewaele, G. Geneste, P. Loubeyre, and M. Mezouar, Phys. Rev. Lett. 113, 265504 (2014).

[25] P. B. Allen and R. C. Dynes, Phys. Rev. B 12, 905 (1975).

[26] A. B. Migdal, Sov. Phys. JETP 34, 996 (1958).

[27] G. M. Eliashberg, Sov. Phys. JETP 11, 696 (1960).

[28] P. B. Allen and B. Mitrović, Theory of Superconducting $T_{c}$, edited by F. S. Henry Ehrenreich and D. Turnbull (Academic Press, 1983), pp. 1-92.

[29] I. Errea, M. Calandra, C. J. Pickard, J. R. Nelson, R. J. Needs, Y. Li, H. Liu, Y. Zhang, Y. Ma, and F. Mauri, Nature (London) 532, 81 (2016).

[30] L. Wang, D. Duan, H. Yu, H. Xie, X. Huang, Y. Ma, F. Tian, D. Li, B. Liu, and T. Cui, Inorg. Chem. 57, 181 (2018).

[31] D. R. Hamann, Phys. Rev. B 88, 085117 (2013).

[32] M. Schlipf and F. Gygi, Comput. Phys. Commun. 196, 36 (2015).

[33] J. P. Perdew, K. Burke, and M. Ernzerhof, Phys. Rev. Lett. 77, 3865 (1996).

[34] P. Giannozzi, S. Baroni, N. Bonini, M. Calandra, R. Car, C. Cavazzoni, D. Ceresoli, G. L. Chiarotti, M. Cococcioni, I. Dabo, A. D. Corso, S. d. Gironcoli, S. Fabris, G. Fratesi, R. Gebauer, U. Gerstmann, C. Gougoussis, A. Kokalj, M. Lazzeri, L. Martin-Samos, N. Marzari, F. Mauri, R. Mazzarello, S. Paolini, A. Pasquarello, L. Paulatto, C. Sbraccia, S. Scandolo, G. Sclauzero, A. P. Seitsonen, A. Smogunov, P. Umari, and R. M. Wentzcovitch, J. Phys. Condens. Matter 21, 395502 (2009).

[35] A. A. Mostofi, J. R. Yates, Y.-S. Lee, I. Souza, D. Vanderbilt, and N. Marzari, Comput. Phys. Commun. 178, 685 (2008).

[36] N. Troullier and J. L. Martins, Phys. Rev. B 43, 1993 (1991).

[37] A. M. Rappe, K. M. Rabe, E. Kaxiras, and J. D. Joannopoulos, Phys. Rev. B 41, 1227 (1990). 